Abdel-Malek, K., Maropis, N., and Yeh, H.J., (1998), "Determining Interference Between Pairs of Solids

Defined Constructively in Computer Animations," Computers in Engineering, v 14 n 11998 p 48-58.

\title{
DETERMINING INTERFERENCE BETWEEN PAIRS OF SOLIDS DEFINED CONSTRUCTIVELY IN COMPUTER ANIMATION
}

\author{
K. Abdel-Malek ${ }^{1}$ \\ Department of Mechanical Engineering and \\ Center for Computer Aided Design \\ The University of Iowa \\ Iowa City, IA 52242 \\ H.J. Yeh \\ Department of Mechanical Engineering and \\ Center for Computer Aided Design \\ The University of Iowa \\ Iowa City, IA 52242
}

\author{
N. Maropis \\ Vice President of Engineering \\ UTI Corporation \\ Collegeville, PA 19426
}

\footnotetext{
${ }^{1}$ Corresponding author
} 


\begin{abstract}
This paper presents theory and implementation of a method for detecting interference between a pair of solid objects. Often times, when performing simulations, two solids may unwittingly interpenetrate each other. The two components of the system presented in this paper are: (1) a surface representation method to model solid objects; and (2) a method for detecting interference. Body representation of a solid in this system is based upon enveloping each solid with surfaces (called positive entities). Most computer aided design CAD systems use solid modeling techniques to represent solid objects. Since most solid models use Boolean operations to model complex objects, a method is presented to envelop complex objects with parametric surfaces. A method for tracing intersection curves between two surfaces is also presented. Discontinuities on surfaces are defined as negative entities in order to extend the method to complex solids. Determining interference is based upon a numerical algorithm for computing points of intersection between boundary curves and parametrized entities. The existence of segments of these curves inside the boundary of positive and negative entities is established by computing the circulation of a function around the boundary curve. Interference between two solids is then detected. No limitations are imposed on the convexity or simplicity of the boundary curves treated.
\end{abstract}

\title{
1 INTRODUCTION
}

A common method of performing simulations is to specify the trajectory of each solid in the simulation environment. In this type of motion-planning, a user specifies a set of constraints on the kinematics of the solid with the time being an additional variable. Thus, it is possible for a solid to unwittingly interfere with a second solid.

A comparative study of the well-known interference algorithms can be found in Geisow and Pratt [1]. A computational method to check for interference of edges and surfaces of two defined solids was presented by Boyse [2]. Pasarello [3] describes a method using Barycentric coordinates and Uchiki et al. [4] uses the space occupancy method. Detecting interference using a characterization of an empty space to describe entities was used by Udupa [5], while Wong and $\mathrm{Fu}$ [6] used a hierarchial orthogonal space to plan a interference-free path. Cameron [7] presented three techniques that can be used when solids are described using the method of constructive solid geometry (CSG). Cameron reports a considerable increase in computational speed over that of classical methods. Other researchers in the field have addressed the real-time interference checking problem by modeling objects as a series of vectors [8], or by modeling solids using convex volumes (spheres) as in Mitsi and Bouzakis [9]. Another type of detecting interference has been approached by minimizing distances between solids in space [10], while Meyer [11] establishes interference by calculating distances between boxes. Gilbert et al. [12] presents a procedure for calculating distances between convex sets in $\mathrm{R}^{\mathrm{m}}$, so that interference can be detected. More recently, Zghal et al. [13] has defined a performance criterion to maximize the shortest distance between two objects. A method using set membership classification was used by Tilove [14] to determine the intersection between two surfaces.

In this paper, a numerical algorithm is presented that determines interference between two moving solids by obtaining static frames of the simulation. The surfaces enveloping each solid are then 
examined for intersection with the surfaces enveloping the other solid. The intersection algorithm is based upon computing the circulation along boundary curves.

\section{SURFACE PARAMETRIZATION}

Solid modeling techniques are used by many CAD systems to model engineering structures and machines (c.f. Akin [15], Requicha [16] and Requicha [17] for a review of solid modeling techniques). A solid modeler typically stores information such as geometry, shape, weight, and material into a database. Complicated solids are modeled using Boolean operators on primitive solids. The method for detecting interference presented in this paper relies upon knowledge of surfaces enveloping the resulting solid. For a solid body A, defined by joining and/or intersecting primitive solids, it is necessary to envelop the resulting solid with a number of parametric surfaces ${ }^{A} \mathbf{x}_{i}(u, v): U \subset R^{2} \rightarrow R^{3},{ }^{A} \mathbf{x}_{i}=\left\{\begin{array}{lll}{ }^{A} \mathbf{x}_{1} & { }^{A} \mathbf{x}_{2} & \ldots\end{array}\right\}$, which is a differentiable map ${ }^{A} \mathbf{x}_{i}$ from an open set $U \subset R^{2}$ into $R^{3}$. That is, the vectors $\partial^{A} \mathbf{x}_{i} / \partial u$ and $\partial^{A} \mathbf{x}_{i} / \partial v$ are linearly independent. This parametrized surface may be bound by either inequality constraints of the form $v_{1} \leq v \leq v_{2}$ and $u_{1} \leq u \leq u_{2}$, or by a bounding closed curve $\mathbf{c}$.

In previous work [25], a simplified method for determining interference between surfaces was presented. That work required the categorization of surfaces into different types of entities. The work presented here is a generalization of the previous work with the addition of a method of determining entities.

In the discussion that follows, it is noted that the bounding curves $\mathbf{c}_{i}$ of those surfaces need be neither convex nor simply connected. The same definition is carried out through the development of the theory for determining interference between pairs of solids.

\subsection{Negative Entities}

In order to model complex mechanical parts, it is necessary to establish a method of representing discontinuities of a surface. A positive entity is defined as a parametrized surface $\mathbf{x}(u, v): U \subset R^{3} \rightarrow S$ bound by a curve $\mathbf{c}_{1}$ (Fig. 1a). A negative entity is defined as a discontinuity on the surface $S$ (a subset of $S$ having the same parametrization $\mathbf{x}$ ) bound by a curve $\mathbf{c}_{2}$, which lies entirely inside the boundary curve $\mathbf{c}_{1}$. Fig. 1b depicts a solid enveloped by 7 entities. Note that negative entity 4 , a region of a circle wrapped around a cylindrical surface, is on the surface of positive entity 2. Note also that the surface of the hole is positive entity 5.

The theory of interference detection presented in the following sections relies upon having a complete representation of entities enveloping solids. The following section presents a method for determining the boundaries of entities enveloping the resulting solid.

\subsection{Enveloping Solids with Surfaces}

To determine surface parametrizations created by performing Boolean operations on solids, it is necessary to find the intersection curve between surfaces enveloping the resulting solid. The 
intersection curves (or disjoint branches of the curve) separate a surface into different entities. Some of these entities are inside the solid while other entities are on the boundary of the resulting solid. Figure 2 illustrates a solid resulting from joining two solids. The intersection curve in Fig. 2 separates solid B into two entities. Entity 2 envelops the resulting solid while entity 1 is internal.

The problem of calculating the intersection curve between two surfaces is one of the most important computational tasks in several engineering applications such as in geometric modeling, CAD/CAM, computer animation, and robotics. Many methods for computing the intersection curves between general surfaces have been derived in the past. Pratt and Geisow [1] summarize and briefly distinguish most of those techniques into five main categories, namely algebraic, lattice evaluation, marching, recursive subdivision, and continuation methods. In this section, only surfaces represented parametrically are treated. A surface is parametrized as

$$
\mathbf{x}^{1}(u, v)
$$

with constrained parameters as

$$
\begin{aligned}
& u_{1} \leq u \leq u_{2} \\
& v_{1} \leq v \leq v_{2}
\end{aligned}
$$

and the second surface is parametrized as

$$
\mathbf{x}^{2}(s, t)
$$

with constrained parameters as

$$
\begin{aligned}
& s_{1} \leq s \leq s_{2} \\
& t_{1} \leq t \leq t_{2}
\end{aligned}
$$

where $(u, v)$ and $(s, t)$ are the independent parametric coordinates. To impose the inequality constraints in numerical form, it is convenient to parametrize the above constraints by introducing new generalized coordinates $\lambda_{i}$ such that an inequality constraint of the form

can be parametrized [20] as

$$
q_{i}^{\text {min }} \leq q_{i} \leq q_{i}^{\max }
$$

where

$$
q_{i}=a_{i}+b_{i} \sin \lambda_{i}
$$

$$
a_{i}=\left(q_{i}{ }^{\max }+q_{i}{ }^{\min }\right) / 2
$$

and

$$
b_{i}=\left(q_{i}^{\max }-q_{i}^{\min }\right) / 2
$$

are the mid-point and half-range of the inequality constraint. In this paper, the computation scheme of the branches of the intersection curve is divided into two phases: (1) finding the starting point and (2) tracing along the curve. The case where a singularity (so-called bifurcation) occurs is also discussed. Tracing a multiple of curves is also addressed by switching the solution using computed tangents.

Finding a Starting Point

For the case of interference of surfaces that results in disjoint branches, it is a nontrivial task to find an initial point on the intersection curve. The technique presented here involves starting from 
an initial guess $\mathbf{q}^{1}$. The intersection problem may be considered as solving the system of seven non-linear equations with eight variables where the constraint function $\mathbf{H}(\mathbf{q})$ is

$$
\mathbf{H}(\mathbf{q})=\left[\begin{array}{c}
\mathbf{x}^{1}(u, v)-\mathbf{x}^{2}(s, t) \\
\left.u-\llbracket\left(u_{1}+u_{2}\right) / 2+\sin \left(\lambda_{1}\right)\left(u_{2}-u_{1}\right) / 2\right] \\
\left.v-\llbracket\left(v_{1}+v_{2}\right) / 2+\sin \left(\lambda_{2}\right)\left(v_{2}-v_{1}\right) / 2\right] \\
s-\left[\left(s_{1}+s_{2}\right) / 2+\sin \left(\lambda_{3}\right)\left(s_{2}-s_{1}\right) / 2\right] \\
t-\left[\left(t_{1}+t_{2}\right) / 2+\sin \left(\lambda_{4}\right)\left(t_{2}-t_{1}\right) / 2\right]
\end{array}\right]=\mathbf{0}
$$

where the inequality constraints of equations (4), (5), (7), and (8) were parametrized per equation (8). The Jacobian of the constraint function $\mathrm{H}(\mathbf{q})$, where $\mathbf{q}=\left\{\begin{array}{llllllll}u & v & s & t & \lambda_{1} & \lambda_{2} & \lambda_{3} & \lambda_{4}\end{array}\right\}$, for a certain initial point $\mathbf{q}^{1}$ is the $3 \times 3$ matrix

$$
\mathbf{H}_{\mathbf{q}}\left(\mathbf{q}^{1}\right)=\left[\frac{\partial \mathbf{H}_{i}}{\partial q_{j}}\left(\mathbf{q}^{1}\right)\right]
$$

To satisfy the constraint equation, an algorithm similar to Newton-Raphson iteration method is employed, as follows:

$$
\mathbf{H}_{\mathbf{q}} \Delta \mathbf{q}=-\mathbf{H}
$$

If the constraint sub-Jacobian matrix is square then equations (11) and (13) comprise the conventional Newton-Raphson iteration method, with its well-known quadratic convergence properties [19]. The constraint equation (equation 11) has more rows than columns and the constraint sub-Jacobian $\mathbf{H}_{\mathbf{q}}$ has more columns than rows, thus equation (13) has multiple solutions. One solution to this type of problems is to find the solution $\Delta \mathbf{q}$ with minimum norm [20] and [21]; i.e., to solve the minimization problem

$$
\begin{gathered}
\min \frac{1}{2} \Delta \mathbf{q}^{T} \Delta \mathbf{q} \\
\mathbf{H}_{\mathbf{q}} \Delta \mathbf{q}=-\mathbf{H}
\end{gathered}
$$

Using the Lagrange multiplier approach that appends a multiplier vector times the equations to be satisfied to the function that is to be minimized, define

$$
\Psi \equiv \frac{1}{2} \Delta \mathbf{q}^{T} \nabla \mathbf{q}-\lambda^{T}\left(\mathbf{H}_{\mathbf{q}} \Delta \mathbf{z}+\mathbf{H}\right)
$$

As a necessary condition for a solution of the minimization problem of equation (13), the gradient of the function in equation (16) is set to zero, yielding

$$
\Delta \mathbf{q}^{T}=\lambda^{T} \mathbf{H}_{\mathbf{q}}
$$

or,

$$
\Delta \mathbf{q}=\mathbf{H}_{\mathbf{q}}^{T} \lambda
$$

Substituting this result into the linearized constraint equations of equation (13) yields

$$
\mathbf{H}_{\mathbf{q}} \mathbf{H}_{\mathbf{q}}^{T} \lambda=-\mathbf{H}
$$

If the sub-Jacobian $\mathbf{H}_{\mathbf{q}}$ has full row rank, then the coefficient matrix on the left of equation (19) is nonsingular. In fact, it is positive definite. Therefore, 


$$
\lambda=\left(\mathbf{H}_{\mathbf{q}} \mathbf{H}_{\mathbf{q}}^{T}\right)^{-1}(-\mathbf{H})
$$

Substituting this result into equation (18) yields the result

$$
\Delta \mathbf{q}=\mathbf{H}_{\mathbf{q}}^{T}\left(\mathbf{H}_{\mathbf{q}} \mathbf{H}_{\mathbf{q}}^{T}\right)^{-1}(-\mathbf{H})
$$

where the coefficient matrix of $(-\mathbf{H})$ is the so-called Moore-Penrose pseudo inverse of the subJacobian. Starting with an initial guess $\mathbf{q}^{1}$, the update of generalized coordinates are calculated through

$$
\Delta \mathbf{q}=\mathbf{H}_{\mathbf{q}}^{*}(-\mathbf{H})
$$

where $\mathbf{H}_{q}^{*}$ is the pseudo-inverse of the Jacobian $\Phi_{q}$, defined by equation (21) as

$$
\mathbf{H}_{\mathbf{q}}^{*}=\mathbf{H}_{\mathbf{q}}^{T}\left(\mathbf{H}_{\mathbf{q}} \mathbf{H}_{\mathbf{q}}^{T}\right)^{-1}
$$

Using this procedure, the starting point $\mathbf{q}^{*}$ on the intersecting curve can be found within a few iterations without adding any new constraint equations. Although this method can quickly converge the initial guess into a starting point, only one starting point will be found, and only the corresponding intersection curve can be traced. If disjoint branches of the curve exist further study of branching from the bifurcation point is necessary.

Tracing along the Curve

Once the starting point is found, the subsequent points on the intersection curve can be traced along the tangent direction. Suppose the parametric coordinates of the starting point $\mathbf{q}^{*}$ are $\left(u^{o}, v^{o}\right)$ and $\left(s^{o}, t^{o}\right)$ with the corresponding $\lambda_{1}^{0}, \lambda_{2}^{0}, \lambda_{3}^{0}$, and $\lambda_{4}^{0}$. The tangent vector $\mathbf{t}\left(\mathbf{H}_{\mathbf{q}}(\mathbf{q})\right)$ to the set defined by $\mathbf{H}(\mathbf{q})=\mathbf{0}$ is uniquely defined by

$$
\mathbf{H}_{\mathbf{q}} \mathbf{t}\left(\mathbf{H}_{\mathbf{q}}\right)=\mathbf{0}
$$

and the normalization

$$
\left\|\mathbf{t}\left(\mathbf{H}_{\mathbf{q}}\right)\right\|_{2}=1
$$

The geometric interpretation of the step marching procedure [19] is shown in Fig. 3 where $\mathrm{c}$ is the step size. The unique tangent vector can be computed at each point along the solution of equation (11) and equation (24) as a basis for proceeding stepwise along the solution curve.

Combining equation (11) and equation (26) yields a system of eight equations in eight variables, such that the Newton-Raphson iteration method can be used to calculate the new point $\mathbf{x}$. By setting the new point as the current point, the tracing is continued until one of two cases occurs: (1) the boundary is reached, or (2) returning to the starting point.

\section{$\underline{\text { Singularity/Bifurcation }}$}

Often times, the curves of intersection (or disjoint branches of the curve) intersect at a point called the bifurcation point. This may occur for a multiple of reasons the most general of which is common tangents. In order to have a complete representation of all intersection curves, it is necessary to detect a bifurcation point along a solution curve (other methods can be found in Keller [22]). In addition, it is required to numerically switch from the curve onto a bifurcating 
branch. This section will consider singular bifurcation points. Treatment of higher order bifurcations can be found in Golubitsky, Stewart, and Scaeffer [23].

Allgower and Georg [19] have showed that for a simple bifurcation point $\mathbf{q}^{\circ}$ of the equation

$$
\mathbf{H}(\mathbf{q})=0
$$

the determinant of the following augmented Jacobian changes sign at the singularity.

$$
\text { sign } \operatorname{det}\left[\begin{array}{c}
\mathbf{H}_{\mathbf{q}} \\
\mathbf{t}\left(\mathbf{H}_{\mathbf{q}}\right)^{T}
\end{array}\right]
$$

where $\mathbf{t}\left(\mathbf{H}_{\mathbf{q}}\right)$ is the tangent function to the set defined by $\mathbf{H}(\mathbf{q})=0$ for $\mathbf{H}: \mathbf{R}^{n} \rightarrow \mathbf{R}^{n-1}$. In practice, equation (27) can be executed by monitoring the Jacobian $\mathbf{H}_{\mathbf{q}}$ for where it becomes row rank deficient of degree one at a bifurcation point $\mathbf{q}^{\circ}$ such that

$$
\operatorname{Rank}\left(\mathbf{H}_{\mathbf{q}}\left(\mathbf{q}^{\circ}\right)\right)=n-2
$$

At such a point, a pair of solution curves pass through the point. Having determined that there is a single bifurcation point, it is of interest to determine this special point. Elementary row matrix operation may be performed to reduce the Jacobian matrix $\mathbf{H}_{\mathbf{q}}$ to the form

$$
\mathbf{E}\left(\mathbf{H}_{\mathbf{q}}\left(\mathbf{q}^{o}\right)\right)=\left[\begin{array}{c}
\mathbf{F}_{\mathbf{q}}\left(\mathbf{q}^{o}\right) \\
\mathbf{0}
\end{array}\right]
$$

where $\mathbf{F}_{\mathbf{q}}\left(\mathbf{q}^{o}\right)$ is an $(n-2) \times n$ matrix with full row rank. Since the transformation $\mathbf{E}$ involves only interchanging equation order, multiplying an equation by a non-zero constant and adding one equation to another, does not change the solution set. Thus equation (11) may be transformed to

$$
\widetilde{\mathbf{H}}(\mathbf{q}) \equiv\left[\begin{array}{l}
\mathbf{F}(\mathbf{q}) \\
\mathbf{f}(\mathbf{q})
\end{array}\right]=\mathbf{E}(\mathbf{H}(\mathbf{q}))=\mathbf{0}
$$

which yields the modified continuation equation as $\widetilde{\mathbf{H}}(\mathbf{q})=\mathbf{0}$. Since only elementary row operations have been used, the upper submatrix on the right is indeed its Jacobian. Since the rank of $\mathbf{F}_{\mathbf{q}}\left(\mathbf{q}^{o}\right)$ was given as (n-2), the null space of $\mathbf{F}_{\mathbf{q}}\left(\mathbf{q}^{o}\right)$ is of dimension two, yielding two nonzero solutions of

$$
\mathbf{F}_{\mathbf{q}}\left(\mathbf{q}^{o}\right) \tau^{i}=\mathbf{0}, \quad i=1,2
$$

where $\tau^{i}$ are tangent vectors which may be orthonormalized such that

$$
\tau^{i^{T}} \tau^{i}=\delta_{i j} \quad i, j=1,2
$$

Thus the objective is to find tangent vectors $\mathbf{t}\left(\mathbf{H}_{\mathbf{q}}\right)$ to continuation curves at $\mathbf{q}^{o}$ of the form

$$
\mathbf{t}\left(\mathrm{H}_{\mathbf{q}}\right)=\sum_{j=1}^{3} \alpha_{j} \tau^{j}=\alpha_{j} \tau^{j}
$$

To be tangent to the solution curve, the equation governing the solution curve should be satisfied to lowest, non-trivial order Taylor approximation [20]; i.e., 


$$
\tilde{\mathrm{H}}\left(\mathbf{q}^{o}+\mathbf{h}\right) \approx \tilde{\mathrm{H}}\left(\mathbf{q}^{o}\right)+\left[\begin{array}{c}
\mathbf{F}\left(\mathbf{q}^{o}\right) \alpha_{j} \tau^{j} \\
\frac{1}{2}\left(\alpha_{l} \tau^{l^{T}}\right) \mathbf{f}_{\mathbf{q q}}\left(\mathbf{q}^{o}\right) \alpha_{k} \tau^{k}
\end{array}\right]=\mathbf{0}
$$

where the Hessian of $\mathbf{f}\left(\mathbf{q}^{o}\right)$ evaluated at $\mathbf{q}^{o}$ is

$$
\mathbf{f}_{\mathbf{q q}}\left(\mathbf{q}^{o}\right) \equiv\left[\frac{\partial^{2} \mathbf{f}\left(\mathbf{q}^{o}\right)}{\partial \mathbf{q}_{i} \partial \mathbf{q}_{j}}\right]
$$

The second order Taylor approximation is required, since its first order Taylor terms are identically zero since $\mathbf{f}_{\mathbf{q}}\left(\mathbf{q}^{o}\right)=\mathbf{0}$.

Since the upper subvector on the right of equation (34) is zero, for all $\alpha_{j}$, equation (34) reduces to

$$
\alpha^{T} \Lambda \alpha=0
$$

where $\alpha=\left[\begin{array}{ll}\alpha_{1} & \alpha_{2}\end{array}\right]^{T}$ and

$$
\Lambda=\left[\tau^{i^{T}} \mathrm{f}_{\mathbf{q q}}\left(\mathbf{q}^{o}\right) \tau^{j}\right]=\left[\Lambda_{i j}\right]
$$

The quadratic equation (equation 36) may be transformed to a quadratic equation in the ratio of the two variables such that

$$
\Lambda_{11}\left(\frac{\alpha_{1}}{\alpha_{2}}\right)^{2}+2 \Lambda_{12}\left(\frac{\alpha_{1}}{\alpha_{2}}\right)+\Lambda_{22}=0
$$

To determine a pair of values for $\alpha_{1}$ and $\alpha_{2}$ that define distinct tangents, the solution is sought such that

$$
\frac{\alpha_{1}}{\alpha_{2}}=-\frac{\Lambda_{12}}{\Lambda_{11}} \pm \frac{1}{\Lambda_{11}} \sqrt{\Lambda_{12}^{2}-\Lambda_{11} \Lambda_{22}} \text { for } \Lambda_{11} \neq 0 \text { and } \Lambda_{22} \neq 0
$$

If $\Lambda_{11}=0$ and $\Lambda_{22}=0$ and $\Lambda_{12} \neq 0$ the solutions are $\alpha^{1}=\left[\begin{array}{ll}1 & 0\end{array}\right]^{T}$ and $\alpha^{2}=\left[\begin{array}{ll}0 & 1\end{array}\right]^{T}$.

In the preceding tracing process, when the trace crosses over the singular point, the determinant of the Jacobian matrix changes signs. In this case, the new tangent of the computed point is inspected to determine whether it orients in opposite direction (within a tolerance) with the previous tangent. If this occurs, the new stepsize must change signs, as shown in Fig. 4a, such that the tracing continues without retracing. Figure $4 \mathrm{~b}$ depicts another situation where the determinant changes signs but the computed tangent will not point in the opposite direction.

Other methods that can be used to detect bifurcation points are presented by Lukacs [24] who proposes an efficient method to detect a first order singularity using eigenvalues. This method can also distinguish whether the tangent point is isolated, an intersection of two branches, passed by only one branch, or is a higher-order singularity.

\section{Numerical Example}

To illustrate the determination of surfaces enveloping a solid using the method outlined above, consider the two surfaces depicted in Fig. 5. 
The equations of these surfaces are given as

$$
\mathbf{x}^{1}(u, v)=\left[\begin{array}{c}
7.07 \cos u \cos v+7.07(\sin u-\sin v)+14.14 \cos v \\
-7.07 \cos u \cos v+7.07(\sin u+\sin v)-14.14 \cos v \\
10 \cos u \sin v+10 \cos v+20 \sin v+50
\end{array}\right]
$$

with inequality constraints defined as

and

$$
\begin{gathered}
0 \leq u \leq 2 \pi \\
-0.785 \leq v \leq 1.57
\end{gathered}
$$

$$
\mathbf{x}^{1}(u, v)=\left[\begin{array}{c}
-7.07 \cos s \cos t+7.07(\sin s+\sin t)-14.14 \cos t \\
-7.07 \cos s \cos t-7.07(\sin s-\sin t)-14.14 \cos t \\
10 \cos s \sin t+10 \cos t+20 \sin t+50 \\
0 \leq s \leq 2 \pi \\
-0.785 \leq t \leq 1.57
\end{array}\right]
$$

The starting point $\mathbf{q}^{*}$ computed using the Moore-Penrose pseudo inverse is

$\mathbf{q}^{*}=\left[\begin{array}{llllllll}0.6184 & 3.4086 & 0.6184 & 2.8745 & 0.1928 & 0.0851 & 0.1928 & -.0851\end{array}\right]$

Using this point as a starting point for the algorithm of Section for mapping continuation curves, a singular bifurcation point is encountered at

$$
\mathbf{q}^{o}=\left[\begin{array}{llllllll}
0.7854 & 3.1416 & 0.7854 & 3.1416 & 0.3398 & 0.8306 \times 10^{-9} & 0.3398 & -0.8306 \times 10^{-9}
\end{array}\right] . \quad \text { The }
$$

tangents computed in the uv-space at the bifurcation point are $\tau^{1}=\left[\begin{array}{ll}0.5773 & 0.8165\end{array}\right]^{T}$ and $\tau^{2}=\left[\begin{array}{ll}-0.5774 & 0.8165\end{array}\right]^{T}$. The resulting intersecting curves in the uv-space are depicted in Fig. 6. There are six surfaces that are partitioned by these curves. Some of these surfaces are internal to the boundary surface of the resulting solid while others are external. The computed intersection curves are shown on the resulting solid in Fig. 7. To illustrate this modeling method, consider the mechanical part shown in Fig. 8.

This solid can be enveloped by positive and negative entities as depicted in Fig. 9. The solid is enveloped by cylindrical surfaces numbered as $1,4,5,12,13,8$, and 9 , and by planar surfaces numbered as 2, 3, 6, 7, 10,11, 14, 15, and 16. Negative entities are: 17 associated with positive entity 7; negative entity 20 associated with positive entity 6; negative entity 18 associated with positive entity 15; and negative entity 19 associated with positive entity 14 . The above 20 entities completely represent the mechanical part.

\section{INTERFERENCE DETECTION}

In the preceding section, it was demonstrated that one can envelop a complex solid with parametric entities. In order to detect collision between two solids, the entities (positive and negative) enveloping the two solids are used to detect interference. The procedure to detect interference between parametric surfaces was presented by Abdel-Malek [25]. In this paper, the interference detection method is generalized. The method relies upon determining intersection points between boundaries of one entity with the surface of another entity and vice versa. The 
curve segments joining these points is then studied for existence inside the boundaries of positive and negative entities by computing the circulation around the bounding curves.

Boundary curves of each entity are checked for interference with the surface of the other entity. The procedure is repeated for each pair of entities associated with each solid. This will result in intersection points on the intersection curve. Curve segments joining these points are then studied for existence inside positive and negative entities. For example, consider the two solids depicted in Fig. 10. Each of the solids is enveloped by a number of entities. The cylindrical entity on each surface has a negative entity associated with it.

To better illustrate the methodology, consider two solids depicted in Fig. 11a, enveloped by planar entities. For two of the surfaces in question, solid 1 has positive entity 1 and negative entity 2, while solid 2 has positive entity 3 and negative entity 4 . Figure $11 \mathrm{~b}$ depicts the two solids at a different instant of time when one is interpenetrating the other.

Entities 1 and 2 are on the surface of the plane $\Omega_{1}$, and entities 3 and 4 are on the plane $\Omega_{2}$. Figure 4 depicts the four entities in more detail. The intersection of the polygonal boundary $\mathbf{c}_{1}(t)$ of entity 1 with the surface $\mathbf{x}^{2}(u, v)$, will result in intersection points $\mathbf{p}_{2}$ and $\mathbf{p}_{8}$. The intersection of the polygonal boundary $\mathbf{c}_{2}(t)$ of entity 2 with the surface $\mathbf{x}^{2}(u, v)$ results in intersection points $\mathbf{p}_{4}$ and $\mathbf{p}_{6}$. Similarly, the intersection of the polygonal boundary $\mathbf{c}_{3}(t)$ with the surface $\mathbf{x}^{1}(u, v)$ results in intersection points $\mathbf{p}_{1}$ and $\mathbf{p}_{7}$. Finally, the intersection of the polygonal boundary $\mathbf{c}_{4}(t)$ with the surface $\mathbf{x}^{1}(u, v)$ results in intersection points $\mathbf{p}_{3}$ and $\mathbf{p}_{5}$.

Thus, the intersection curve is fully defined; i.e., all boundary points of intersection are computed. A necessary condition for the two solids in Fig. 11b to collide is that a line segment joining two consecutive points of intersection, coexists inside boundaries of positive entities, but outside boundaries of negative entities. Since all points of intersection are computed, it is possible to represent each line segment on the line of intersection by a single point $\mathbf{s}=\left(\mathbf{p}_{i+1}+\mathbf{p}_{i}\right) / 2$, for $i=$ $1, \ldots, 7$. To study the existence of a point inside a boundary $\mathbf{c}$, let $\zeta(\mathbf{s})$ be the indicator [25] for a point $\mathbf{s}$ which determines its existence inside the boundary $\mathbf{c}(t)$ of a positive entity, such that

$$
\zeta(\mathbf{s})=\frac{1}{2 \pi} \oint_{\mathbf{c}} d \tan ^{-1}\left(\frac{y-s_{y}}{x-s_{x}}\right)=\oint_{\mathbf{c}} d \theta=\left\{\begin{array}{cc}
1 & \text { Inside boundary } \mathbf{c}(t) \\
0 & \text { Outside boundary } \mathbf{c}(t)
\end{array}\right.
$$

where $d \theta$ is the change in angle swept by a vector subtended from the point $\mathbf{s}$ to the boundary $\mathbf{c}(t)$, as depicted in Figs. 13a and 13b, inside and outside respectively.

\section{Lemma 1}

A point $\mathbf{s}$ is outside the boundary $\mathbf{c}$ if and only if it satisfies the following equation

$$
\zeta(\mathbf{s})=0
$$

$\underline{\text { Proof }}$ 
Proof of equation (43) is presented for a point $\mathbf{s}$ outside the boundary $\mathbf{c}(\mathrm{t})$. If there exists a potential function $\mathbf{F}$, continuous for all points inside the closed curve $\mathbf{c}(\mathrm{t})$, such that $\nabla \times \mathbf{F}=0$ in the region, then $\mathbf{F}$ is the gradient of a single valued scalar function $\phi$ in that region $\mathbf{F}=\nabla \phi$, and the circulation of $\mathbf{F}$ around $\mathbf{c}(\mathrm{t})$ is zero, i.e.,

$$
\oint_{\mathbf{c}} \mathbf{F} \cdot d \mathbf{r}=\oint_{\mathbf{c}} d \phi=0
$$

Consider a potential function $\mathbf{F}$,

$$
\mathbf{F}=\left[\begin{array}{c}
-\frac{(\mathbf{p}-\mathbf{s}) \bullet \mathbf{j}}{|\mathbf{p}-\mathbf{s}|^{2}} \\
\frac{(\mathbf{p}-\mathbf{s}) \cdot \mathbf{i}}{|\mathbf{p}-\mathbf{s}|^{2}}
\end{array}\right]=\left[\begin{array}{c}
-\frac{y-s_{y}}{\left(x-s_{x}\right)^{2}+\left(y-s_{y}\right)^{2}} \\
\frac{x-s_{x}}{\left(x-s_{x}\right)^{2}+\left(y-s_{y}\right)^{2}}
\end{array}\right]
$$

$\mathbf{F}$ is continuous in the region bound by $\mathbf{c}$ ( since the point $\mathbf{s}$ is outside), $\mathbf{p}=\left[\begin{array}{ll}x & y\end{array}\right]^{T}$ is any point on the curve $\mathbf{c}$, and $\mathbf{s}=\left[\begin{array}{ll}s_{x} & s_{y}\end{array}\right]^{T}$. Then,

$$
\begin{gathered}
\mathbf{F} \bullet d \mathbf{r}=\frac{\left(x-s_{x}\right) d y-\left(y-s_{y}\right) d x}{\left(x-s_{x}\right)^{2}+\left(y-s_{y}\right)^{2}} \\
=d \tan ^{-1}\left(\frac{y-s_{y}}{x-s_{x}}\right)=d \varphi
\end{gathered}
$$

Thus, for a closed curve $\boldsymbol{c}$, the circulation of $\mathbf{F}$ around $\mathbf{c}$ is zero, which indicates that the change of angle for a point outside the closed curve $\mathbf{c}$ is zero.

\section{Lemma 2}

A point $\mathbf{s}$ is inside the boundary $\mathbf{c}$ if and only if it satisfies the

$$
\zeta(\mathbf{s})-1=0
$$

Proof

To prove that the change of angle for a point inside the closed curve $\mathbf{c}$ is equal to $2 \pi$, Cauchy's residue theorem is used [26]. If the point $\mathbf{s}$ is inside the region bound by the closed curve $\mathbf{c}$, a singularity occurs for the function $\mathbf{F}$ at the point $\mathbf{s}$. The residue theorem states that if a singularity exists, then

$$
\oint_{\mathbf{c}} f(z) d z=2 \pi i \sum_{k=1}^{n} \operatorname{Res}\left(a_{k}\right)
$$

Where $a_{k}$ are the poles of the function inside the region. Thus for the integral $\oint_{c} d \theta$, let

$$
z-s=e^{i \theta}
$$

differentiating both sides of equation (50), we get

$$
d \theta=\frac{d z}{i(z-s)}
$$

integrating equation (51),

$$
\oint_{\mathbf{c}} d \theta=\oint_{\mathbf{c}} \frac{d z}{i(z-s)}=2 \pi i\left(\lim _{z \rightarrow p} \frac{z-p}{i(z-p)}\right)=2 \pi
$$


i.e., for a point inside a closed region, the change of angle experienced is $2 \pi$.

In stating equation (42), constraints have not been made on the complexity of the curve. The bounding curve need not be simple nor convex as illustrated (without proof) in Fig. 14. In the nonsimple case, the ray encounters $n$ loops. The indicator has value one only in the loop that is surrounding the point $\mathbf{s}$. Figure 14a depicts two loops, thus, the indicator will determine that the point $\mathbf{s}$ is inside the curve $\mathbf{c}$. In the case of a nonconvex curve, the value of the swept angle will increase from $\theta_{1}$ to $\theta_{2}$, then decrease again $\theta_{3}$ as depicted in Fig. 14b. The total change in angle will still be $2 \pi$ for a point inside and zero for a point outside.

To determine whether interference occurs between a pair of type II entities, it is necessary to establish whether the point $\mathbf{s}$, exists inside all positive and negative entities associated with the two surfaces. Let $\zeta_{1 . . n}$ be the indicator for this purpose, where $\mathrm{n}$ is the total number of entities.

\section{Lemma 3}

The indicator between two surfaces having a multiple of positive and negative entities is

$$
\zeta_{1 . n}=\prod_{k=1}^{m} \zeta_{k} \prod_{l=m+1}^{n}\left(1-\zeta_{l}\right)=\left\{\begin{array}{cc}
1 & \text { point inside } \\
0 & \text { point outside }
\end{array}\right.
$$

where $\zeta$ is defined in equation (42)

Proof

For two positive entities a and $b$, interference will occur if and only if the line segments lay inside the boundaries of both entities. The combined indicator for two positive entities is

$$
\zeta_{a b}(\mathbf{s})=\zeta_{a}(\mathbf{s}) \zeta_{b}(\mathbf{s})
$$

where $\zeta_{a}(\mathbf{s})$ is the indicator for entity a, $\zeta_{b}(\mathbf{s})$ is the indicator for entity b, and $\zeta_{a b}(\mathbf{s})$ is the combined indicator. In case of negative entities, the indicator for a negative entity (e.g., entity 2 ) is defined as

$$
\zeta_{2 \text { negative }}(\mathbf{s})=1-\frac{1}{2 \pi} \oint_{\mathbf{c}} d \theta=1-\zeta_{2}(\mathbf{s})= \begin{cases}1 & \text { point outside } \\ 0 & \text { point inside }\end{cases}
$$

For example, the combined indicator for the line segment on the intersection curve in Fig. 12, represented by a point $\mathbf{s}$, is

$$
\zeta_{1234}(\mathbf{s})=\zeta_{1}(\mathbf{s})\left(1-\zeta_{2}(\mathbf{s})\right) \zeta_{3}(\mathbf{s})\left(1-\zeta_{4}(\mathbf{s})\right)
$$

Thus for two surfaces comprising positive and negative entities, the indicator can be written as

$$
\zeta_{1 . . . n}=\prod_{k=1}^{m} \zeta_{k} \prod_{l=m+1}^{n}\left(1-\zeta_{l}\right)
$$

where $m$ is the number of positive entities, and $n-m$ is the number of negative entities on the two surfaces. Consider the middle point $\mathbf{s}_{1}$ on line segment $\overline{\mathbf{p}_{1} \mathbf{p}_{2}}$ in Fig. 12. The point is outside boundaries $\mathbf{c}_{1}(t), \mathbf{c}_{2}(t)$ and $\mathbf{c}_{4}(t)$ thus $\zeta_{1}\left(\mathbf{s}_{1}\right)=\zeta_{2}\left(\mathbf{s}_{1}\right)=\zeta_{4}\left(\mathbf{s}_{1}\right)=0$, and inside boundary $\mathbf{c}_{3}(t)$ thus $\zeta_{3}(\mathbf{s})=1$. Using equation (53), the combined indicator $\zeta_{1234}\left(\mathbf{s}_{1}\right)=0$ indicates no interference due to this segment. While the middle point $\mathbf{s}_{2}$ on line segment $\overline{\mathbf{p}_{2} \mathbf{p}_{3}}$ has 
$\zeta_{1}\left(\mathbf{s}_{2}\right)=\zeta_{3}\left(\mathbf{s}_{2}\right)=1$, and $\zeta_{2}\left(\mathbf{s}_{2}\right)=\zeta_{4}\left(\mathbf{s}_{2}\right)=0$ which leads to $\zeta_{1234}\left(\mathbf{s}_{2}\right)=1$ indicating interference of the two solids.

The problem of interference checking of solids is reduced to one of determining whether a segment of a curve (represented by a point $s$ ) is inside entity boundaries. To solve this problem, Boyse [2] suggests casting a ray from the point $s$ to infinity in the plane. The ray intersects the boundaries of the so-called face plane (Fig. 15a depicts a face having a discontinuity). If the ray intersects the edges an odd number of times then the point $s$ is inside the face boundaries (Fig. $15 \mathrm{~b}$ ). If the ray intersects the edges an even number of times then the point is outside. This method has many deficiencies and is difficult to implement on a computer. It necessitates breaking the area into simpler polygonal surfaces and thus eliminating discontinuities on the surface (the so-called bridging method). Another deficiency arises when the ray coincides with an edge, thus having an infinite number of intersections.

If the boundary is composed of a multiple of curves (e.g., lines and arcs), then the region is segmented into a number of entities. Consider two solids, solid A is enveloped by surfaces parametrized as ${ }^{A} \mathbf{x}^{i}(u, v)$ and each surface having a boundary curve ${ }^{A} \mathbf{c}^{j}(w)$. Solid $\mathrm{B}$ is enveloped by surfaces parametrized as ${ }^{B} \mathbf{x}^{i}(s, t)$ and have boundary curves ${ }^{B} \mathbf{c}^{j}(w)$. The points on the intersection curve are the solution set to $\Lambda$ where

$$
\Lambda=\left[\begin{array}{c}
{ }^{A} \mathbf{x}^{i}(u, v)-{ }^{B} \mathbf{c}^{j}(w) \\
{ }^{A} \mathbf{c}^{j}(w)-{ }^{B} \mathbf{x}^{i}(s, t)
\end{array}\right]=\mathbf{0}
$$

The solution set of $\Lambda$ is ordered such that the points repositioned. Interference between the two solids will occur if and only if the middle point on the curve joining each two consecutive points of the solution set satisfies equation (57).

\section{CONCLUSIONS}

A method to detect interference between two solids is presented. To use this method, it is necessary to obtain parametric representations of the surfaces enveloping the solid. It has been shown that to determine these surfaces, it is necessary to determine the intersection curves between the surfaces. It was also shown that in many cases, intersection curves meet at a specific point called the bifurcation point. To numerically compute all branches of the intersection curves, tangents have to be computed in the space of the parametric variables. A continuation method is then used to trace each curve.

The surfaces computed are then used to detect interference between a pair of solid bodies during a simulation or animation. The numerical algorithm is valid for complex solids due to the ability of representing solids with holes. Mechanical parts are represented with a high degree of fidelity. The algorithm, however, is computationally intensive.

\section{REFERENCES}


1. Pratt, M.J.; and Geisow, A.D. (1986) Surface/Surface Intersection Problems, The Mathematics of Surfaces, (Gregory, J.A., editor), Clarendon Press, Oxford, 6:117-142.

2. Boyse, J.W. (1979), Interference Detection Among Solids and Surfaces, Communications of ACM, v.22, no.1, January 1979, pp. 3-9.

3. Pasarello, C.E. (1982), Interference Detection Using Barycentric Coordinates, Mechanics Research Communications (UK), v.9, no.6, pp.373-378.

4. Uchiki, T.; Ohashi, T.; and Tokoro, M. (1983), Collision Detection in Motion Simulations. Computer and Graphics, vol. 7, no. 3-4, pp. 285-293.

5. Udupa, S.M. (1977) Collision Detection and Avoidance in Computer Controlled Manipulators. Proceedings of the 5th IJCAI-Robotics, pp. 737-748.

6. Wong, S.; Fu, K.S. (1985) A Hierarchial Orthogonal Space Approach to Collision Free Path Planning. IEEE no. 7, pp. 506-511.

7. Cameron, S. (1989) Efficient Intersection Tests for Objects Defined Constructively. Int. J. of Robot. Res., 8(1):3-25

8. Seitz, B.; Cipra, R.J. (1992) Real-time Collision Avoidance of a Planar Manipulator with an Interfering Single Link Arm. Proceedings of the IEEE International Conference on Robotics and Automation, v.2, pp. 1494-1499.

9. Mitsi, S.; Bouzakis, K. (1993) Simulation of Redundant Manipulators for Collision Avoidance in Manufacturing and Assembly Environments. Mechanism and Machine Theory, v.28, No. 1, pp. 13-21.

10. Wang, D.; Hamam, Y. (1992) Optimal Trajectory Planning of Manipulators with Collision Detection and Avoidance. Int. J. of Robotics Research, v. 11, n. 5, pp. 460-468.

11. Meyer, W. (1986) Distances Between Boxes: Applications to Collision Detection and Clipping. IEEE International Conference on Robotics and Automation, pp.597-602.

12. Gilbert, E.G. 1989, "Computing the Distance Between Smooth Objects in Three-Dimensional Space," Proc. of IEEE Int. Conf. Rob. Autom., Scottsdale, AZ, Vol. I, pp. 158-163.

13. Zghal, H., et al. (1992) Collision Avoidance of a Multiple Degree of Redundandancy Manipulator Operating Through a Window. J. of Dynamic Systems, v.114, no.4, pp. 717721.

14. Tilove, R.B. (1980) Set Membership Classification: A Unified Approach to Geometric Intersection Problems. IEEE Trans. on Computers, 29(10):874-883. 
15. Akin, J.E. (1990) Computer Assisted Mechanical Design, Prentice Hall, Englewood Cliffs, New Jersey.

16. Requicha, A.A. (1980) Representations for Rigid Solids: Theory, Methods, and Systems. Computing Surveys, v.12, no.14, p.437.

17. Requicha, A.A. (1983) Solid Modeling: Current Status and Research Directions. IEEE Computer Graphics and Applications, Vol. 3, No. 7, pp. 25-30 and 32-37.

18. Qiulin, D.; Davies, B.J. (1987) Surface Engineering Geometry for Computer-Aided Design and Manufactureing, Ellis Horwood, Chichester, U.K.

19. Allgower, E.L.; Georg, K. (1990) Numerical Continuation Methods, Springer-Verlag, Berlin, Hedelberg.

20. Haug, E.J.; Luh, C.M.; Adkins, F.; Wang, J.Y. (1994) Numerical Algorithms for Mapping Boundaries of Manipulator Workspaces. Proceedings of the 24th ASME Mechansims Conference.

21. Noble, B., and Daniel, J.W. (1988) Applied Linear Algebra, Prentice-Hall, Englewood Cliffs, NJ.

22. Keller, H.B. (1987) Lectures on Numerical Methods in Bifurcation Problems. Springer Verlag, Berlin, Heidelberg, New York.

23. Golubitsky, M.; Stewart, I.; and Schaeffer, D.G. (1988) Singularities and Groups in Bifurcation Theory. Vol II, Springer Verlag, Berlin, Heidelberg, New York.

24. Lucaks, G. (1990) Simple Singularities in Surface-Surface Intersections. The Mathematics of Surfaces, (ed. Gregory, J.A.), Clarendon Press, Oxford.

25. Abdelmalek, K. (1995) A Numerical Algorithm for Detecting Interference Among Complex Links of Manipulator Arms. To appear in Int. J. of Robotics and Automation.

26. Hildebrand, F.B. (1976) Advanced Calculus For Applications, Prentice Hall, Englewood Cliffs, NJ.

\section{LEGEND TO FIGURES}

Fig. 1. (a) Positive and negative entities, (b) a surface parametrization of a solid model

Fig. 2. A solid resulting from the union of two solids

Fig. 3. The step marching procedure for tracing the curve 
Fig. 4. (a) Tangents in opposite directions (b) tangent will not point in opposite directions Fig. 5. Two solids combined into one solid

Fig. 6. Computed intersection curves in the uv-space

Fig. 7. Intersection curves depicted on the combined solid

Fig. 8. A mechanical part

Fig. 9. Entities enveloping a mechanical part

Fig. 10. Two solids in interference

Fig. 11. (a) Two solids having positive and negative entities (b) Two solids in interference

Fig. 12. Determining intersection points

Fig. 13. A vector sweeping the boundary with center at $\mathbf{s}$; (a) inside c, and (b) outside

Fig. 14. (a) A point inside a nonsimple curve (b) a point inside a nonconvex curve

Fig. 15. (a) An entity having a discontinuity, (b) the bridging method and casting a ray 


\section{Illustrations}

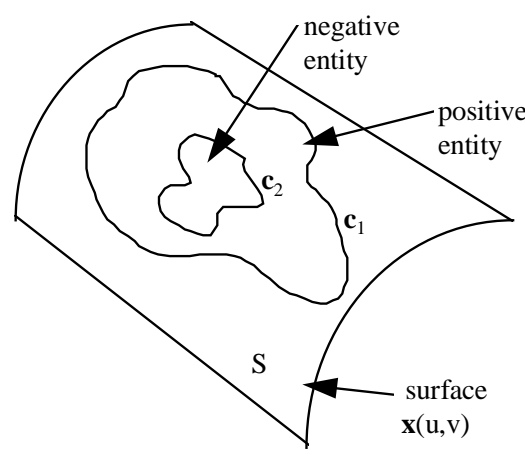

(a)

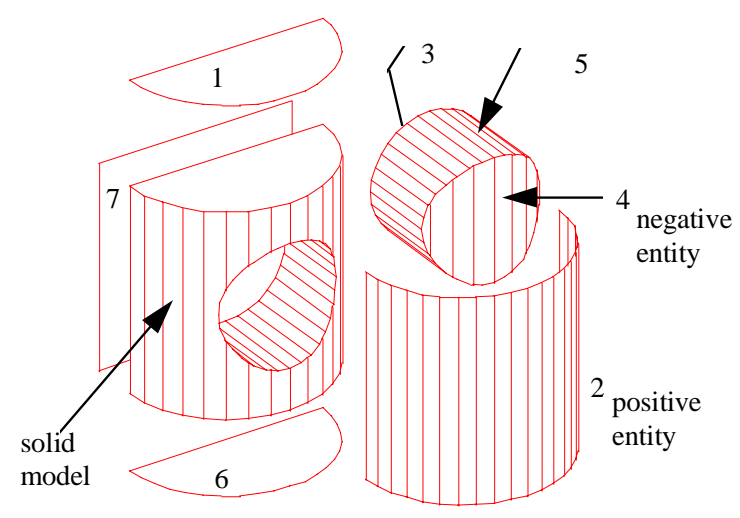

(b)

Fig. 1. (a) Positive and negative entities, (b) a surface parametrization of a solid model

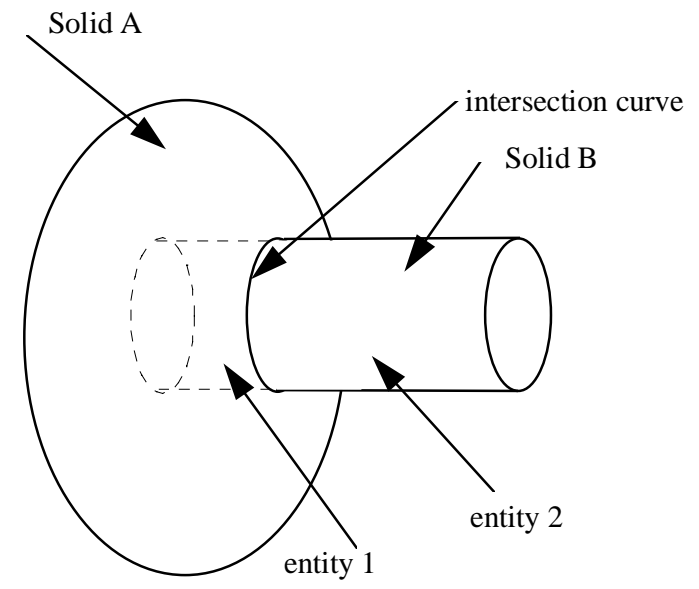

Fig. 2. A solid resulting from the union of two solids 


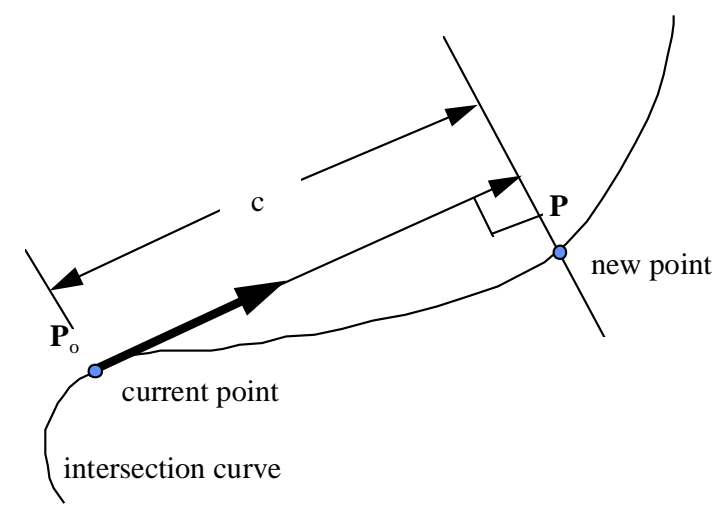

Fig. 3. The step marching procedure for tracing the curve

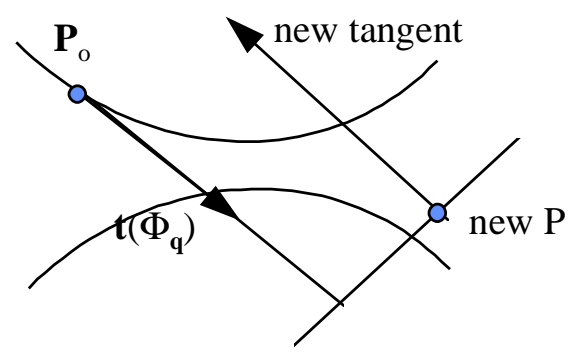

(a)

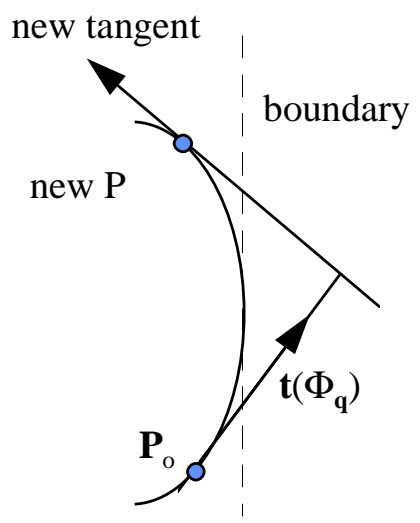

(b)

Fig. 4. (a) Tangents in opposite directions (b) tangent will not point in opposite directions 
Fig. 5. Two parametric surfaces

Fig. 6. Computed intersection curves in the uv-space

Fig. 7. Intersection curves depicted on the combined solid 


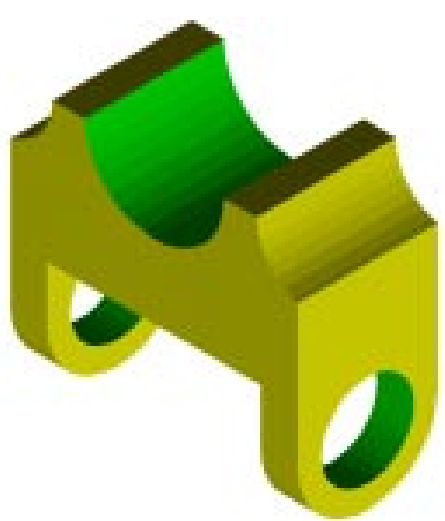

Fig. 8. A mechanical part

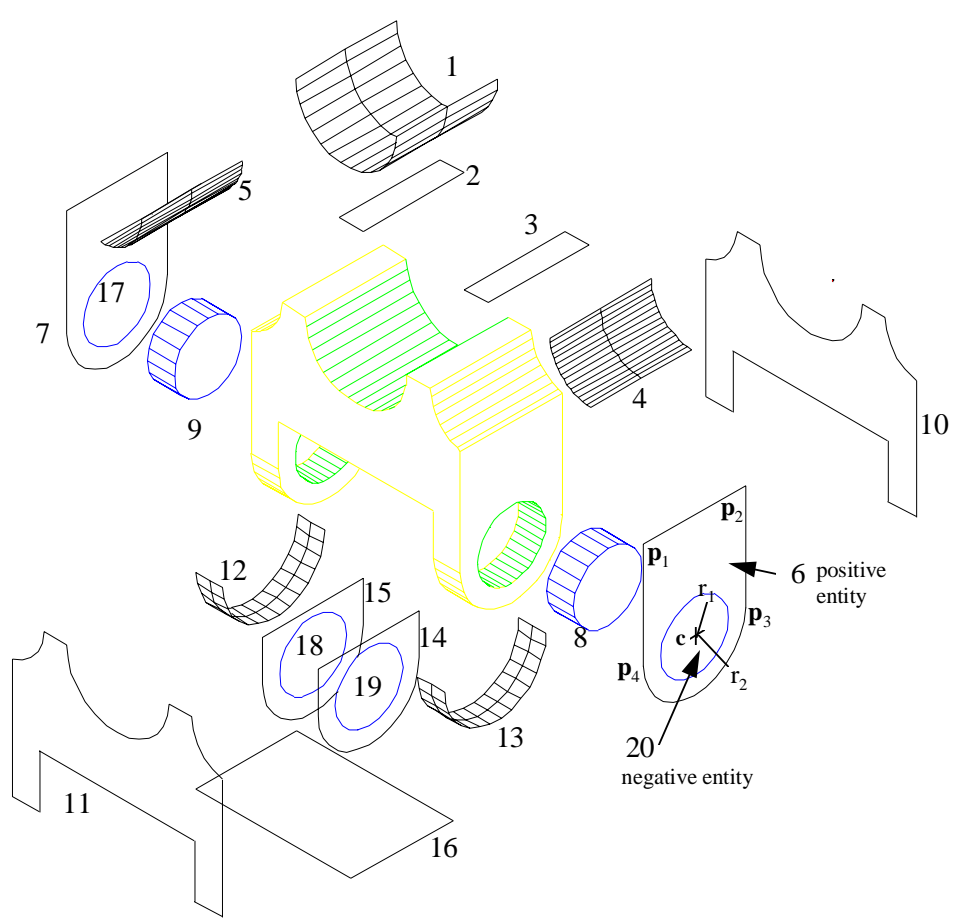

Fig. 9. Entities enveloping a mechanical part 


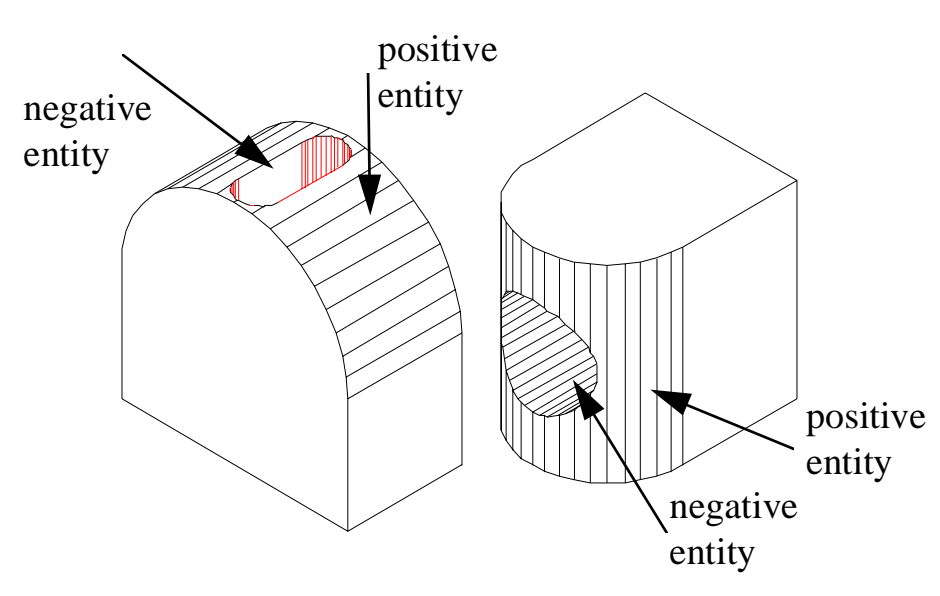

(a)

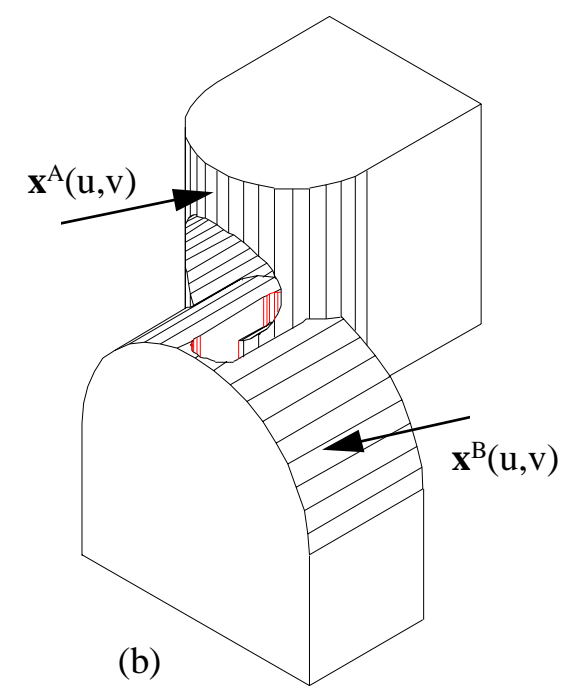

(b)

Fig. 10. Two solids in interference

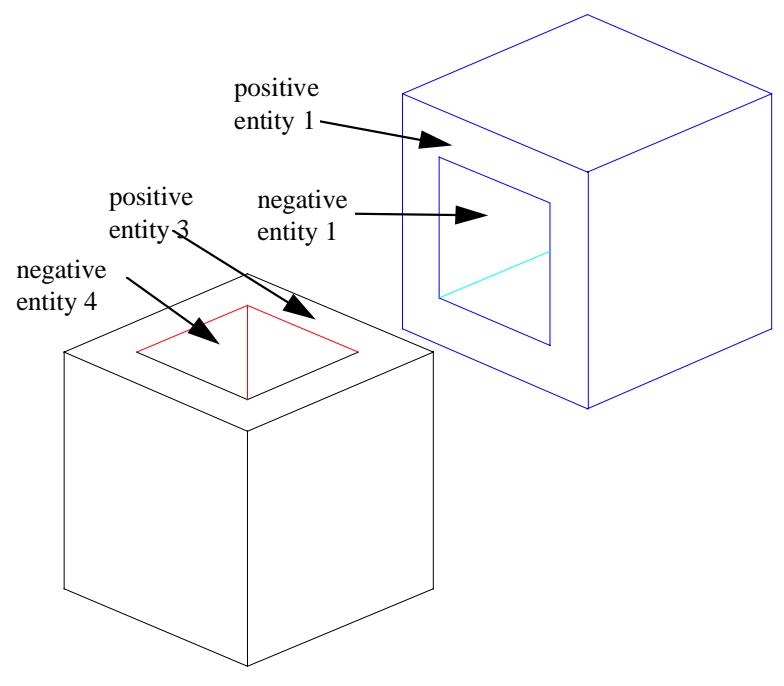

(a)

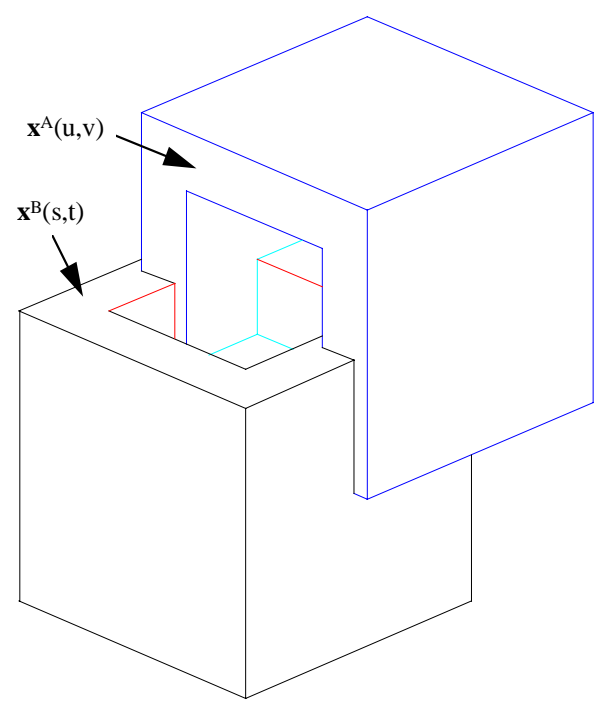

(b)

Fig. 11. (a) Two solids having positive and negative entities

(b) Two solids in interference 


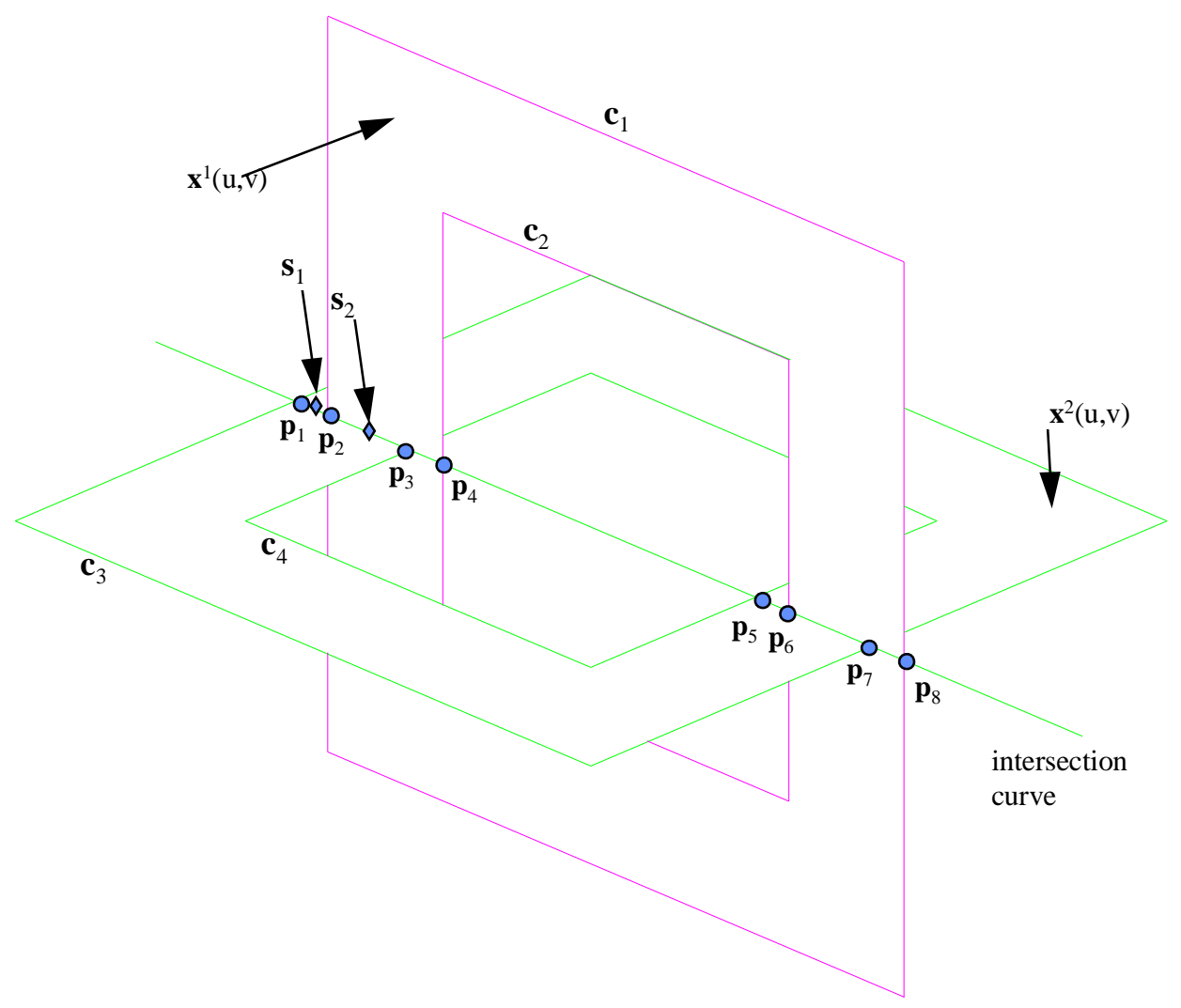

Fig. 12. Determining intersection points

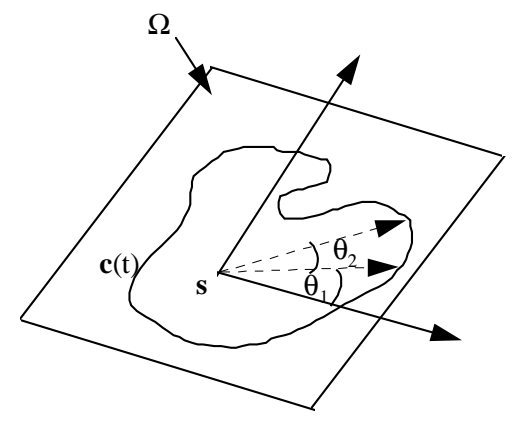

(a)

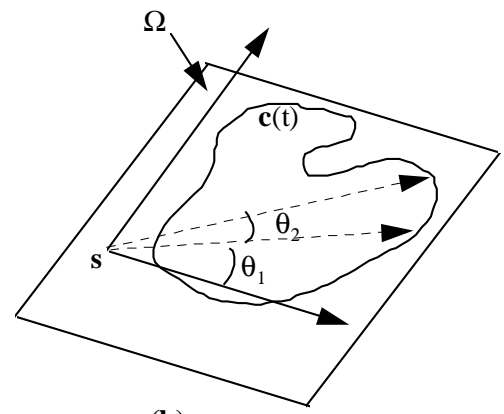

(b)

Fig. 13. A vector sweeping the boundary with center at $\mathbf{s}$; (a) inside $\mathbf{c}$, and (b) outside $\mathbf{c}$ 


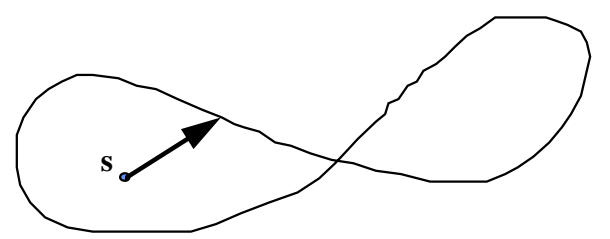

(a)

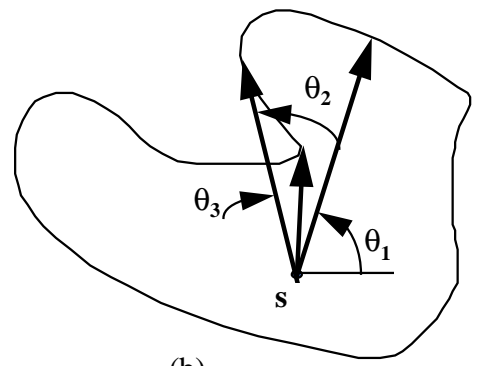

(b)

Fig. 14. (a) A point inside a nonsimple curve (b) a point inside a nonconvex curve
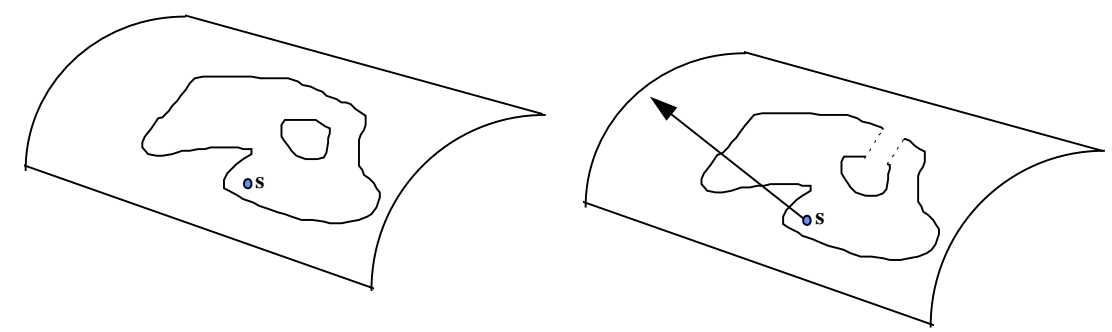

Fig. 15. (a) An entity having a discontinuity, (b) the bridging method and casting a ray 